\title{
Compromised immune response to viral infections in type 2 diabetes mellitus
}

\author{
Sudhir Kumar Ambati* \\ Department of Epidemiology, University of Medicine and Health Science, St. Kitts and Nevis
}

Received: 15 October 2020

Revised: 03 November 2020

Accepted: 04 November 2020

\section{*Correspondence:}

Dr. Sudhir Kumar Ambati,

E-mail: sudhirambati@gmail.com

Copyright: $\odot$ the author(s), publisher and licensee Medip Academy. This is an open-access article distributed under the terms of the Creative Commons Attribution Non-Commercial License, which permits unrestricted non-commercial use, distribution, and reproduction in any medium, provided the original work is properly cited.

\begin{abstract}
Viral infections are common in people with type 2 diabetes, which is associated with poor immune responses. In type 2 diabetes, the normal functioning of the immune system and immune cells is suppressed, which allows the viruses to enter the host cells and cause infections. The impacts of type 2 diabetes on immune response and viral infections are associated with different mechanisms, including suppression or impairment of cytokine production, dysfunctioning of immune cells, defects in the phagocytosis, and disruption of natural killer cells. The high concentration of blood glucose or hyperglycemia in type 2 diabetes affects the production of cytokines, including interleukins, interferon, TNF- $\alpha$, and IFN $-\gamma$. These cytokines are involved in fighting pathogens, and they induce the production of antibodies involved in the adaptive immune response. Therefore, disruption of these cytokines production leads to compromised immune response and invading pathogens like viruses enter and replicate in the host body.
\end{abstract}

Keywords: Type 2 diabetes mellitus, Immune response, Cytokines, Viral infection

\section{INTRODUCTION}

The increasing prevalence rate of type 2 diabetes mellitus has been the main cause of death globally. The existing body of evidence from the world health organization (WHO) demonstrates that nearly 422 million people worldwide are affected by diabetes mellitus. ${ }^{1}$ Between 2000 and 2016, there was a 5\% increase in premature mortality from diabetes. ${ }^{1}$ Type 2 diabetes is known to affect the immune systems.

The innate and adaptive immune factors are the essential etiological components involved in the occurrence of insulin resistance. The abnormal proliferation of innate and adaptive immune factors occurs due to inflammation of adipose tissues that results in the onset of type 2 diabetes. ${ }^{2}$ It is apparent that in patients with uncontrolled diabetes, the immune system is altered, which increases the susceptibility to viral or bacterial infections. ${ }^{1}$ There is also an altered immune response due to elevated concentrations of glucose that contributes to the production of advanced glycation end-products and constant adipose tissue inflammation. ${ }^{1}$

The compromised immune response is common in people with poorly controlled diabetes due to poor glycemic control, which is powerfully related to serious infections. The extant literature indicates that viral infections are among the most common infections in people with type 2 diabetes. ${ }^{3}$ These patients have increased risks of viral infections with viruses like Kaposi's sarcoma-associated herpesvirus (KSHV or HHV-8), West Nile virus encephalitis virus, severe acute respiratory syndrome coronavirus (SARS-CoV), middle east respiratory syndrome coronavirus (MERS-CoV), SARS coronavirus 2 (SARS-CoV-2), and hepatitis $\mathrm{C}$ virus. The most current viral infection associated with compromised immune response in people with type 2 diabetes is COVID-19 
infections caused by the SARS-CoV-2 virus. ${ }^{3}$ This study thus seeks to provide evidence on the impacts of type 2 diabetes mellitus on the immune system and how it compromises the immune response to viral infections. The study aims to address the following research question; how does type 2 diabetes impact the immune system in our body and compromise immune response to viral infections?

\section{Type 2 diabetes mellitus and immune response}

Under normal circumstances, the human body uses protection mechanisms against the invasion of different viruses, parasites, bacteria, fungi, and toxins. These pathogens do not penetrate the defence system, but several defects contribute to the immune system's poor functioning. ${ }^{4}$ Under such conditions or defects, the pathogenic invasion is high where they can cause infections. The defects are associated with poor immune response, especially in type 2 diabetes mellitus.

In people diagnosed with type 2 diabetes, the immune response is compromised or disrupted due to damages of natural barriers like mucosal surfaces and hyperglycemia. ${ }^{4}$ According to the American diabetes association, poor immune response is attributed to the failure of the immune system to fight off the invasion of pathogens. ${ }^{5}$ Diabetes related mechanisms that compromise the immune response to pathogenic infections include suppression or impairment of cytokine production, dysfunctioning of immune cells, defects in the phagocytosis, and failure to kill pathogens. ${ }^{4}$

Cytokines like interleukins (IL) play a crucial role in altering immune response in people with type 2 diabetes mellitus. High glucose concentrations in type 2 diabetes suppress cytokines production, mainly IL-2, IL-6, and IL10. ${ }^{5}$ IL-6, have a crucial role in protecting against pathogens and inducing the production of antibodies that promote an adaptive immune response. In type 2 diabetes, these cytokines are inhibited, which in turn suppresses immune response to the invading pathogens. ${ }^{4,5}$ Besides, other cytokines like interferon-gamma (IFN- $\gamma$ ) and tumor necrosis factor-alpha (TNF- $\alpha$ ) also lead to disrupted or compromised immune response in type 2 diabetes. The existing body of literature indicated that high glucose concentrations in type 2 diabetes affect T-cell's normal functions, which leads to reduce the production of IFN- $\gamma$ and TNF- $\alpha{ }^{6}$ This reduces viral or bacterial load in PBMCs that impairs immune cell's capacity to control the growth of pathogens during an infection. ${ }^{7,8}$ Dysfunction of immune cells is also a risk factor for compromised immune response in type 2 diabetes.

Resistin is a peptide hormone from adipose tissue and is involved in resistance to insulin. In patients with type 2 diabetes, these resistin levels are increased. ${ }^{9}$ An increase in resistin levels reduces reactive oxygen species (ROS) production in neutrophils and suppresses neutrophil extracellular traps due to neutrophil degranulation and dysfunction. Such dysfunctions and impairment in neutrophils are associated with high glucose concentrations that increase pathogenic infection susceptibility. ${ }^{10}$ Similarly, macrophages play a crucial role in altered immune response due to reduced phagocytosis.

Type 2 diabetes is associated with hyperglycemia that alters macrophage's normal functions and causes a compromised immune response. High levels of glucose in type 2 diabetes affect the function of macrophages. ${ }^{11}$ High glucose levels in type 2 diabetes mellitus leads to defects in macrophages that lead to phagocytosis impairment. Such impairment occurs due to reduced glycolytic capacity, which is associated with long-term sensitization to high glucose levels. ${ }^{11}$ Further, high levels of glucose increase M2 macrophage markers, including Arginase 1 and IL-10. Based on the fact that the microbicidal capacity of M2 macrophages is poor, higher glucose levels in type 2 diabetes negatively impacts the macrophages markers that weaken the immune response against pathogenic infections. ${ }^{12}$ Besides, failure to kill pathogens indicates poor immune response.

Natural killer cells, which play a crucial role in controlling pathogen invasion, are involved in compromised immune response in people with type 2 diabetes. ${ }^{13}$ The researchers found that in type 2 diabetes mellitus, the natural killer cells are affected by high glucose levels. ${ }^{13}$ High levels of glucose cause defects in the natural killer cell-activating receptors called NKG2D and NKp46 due to the degranulation capacity of natural killer cells. The defects in such receptors weaken the natural killer cells, which in turn, alters the immune response to invading pathogens. ${ }^{13}$ The defence mechanisms or innate and adaptive-humoral or cellular immune systems do not function properly because of altered immune cells.

Viral infections in type 2 diabetes mellitus are associated with the impairment of beta cells, insulin resistance, and glucose metabolism. The existing body of evidence demonstrates that people with type 2 diabetes mellitus have impaired beta-cell responsiveness, abnormal blood glucose metabolism, and insulin resistance. ${ }^{14}$ The primary mechanism of viral infections in type 2 diabetes entails persistent infections of beta cells in the pancreas, which provokes cell damage. In addition, pancreatic beta cells infections enhance the release of sequestered antigens, which induces autoimmune response against viruses. ${ }^{14}$ Antiviral immune response is also compromised in type 2 diabetes mellitus due to delayed induction of cytokines and viral replication. A study that examined how type 2 diabetes mellitus alters viral immune response in relation to West Nile virus infection shows cytokines role in enhancing the infection. ${ }^{15}$ The study revealed that type 2 diabetes damages natural barriers, which reduce the release of cytokines, mainly IFN- $\alpha$. The study revealed that damage to the natural barriers leads to compromised antiviral immune response, characterized by delayed 
IFN- $\alpha$ induction and reduced IgM and IgG antibodies. ${ }^{15}$ Reduced levels of antibodies thus make the body unable to fight pathogen invasion and facilitate the replication of West Nile virus in the body. ${ }^{15}$

\section{Overactive inflammatory responses in type 2 diabetes mellitus}

The impacts of type 2 diabetes on immune response and viral infections are also associated with over activated inflammatory responses associated with damage to essential organs in the body systems. ${ }^{3}$

The over activated inflammatory responses have been witnessed in the SARS-CoV-2 virus that causes COVID19 infections in people with type 2 diabetes. It is apparent that in diabetes mellitus, the levels of inflammatory markers, such as IL-6, C-reactive protein (CRP), troponin, lactate dehydrogenase $(\mathrm{LDH})$, and $\mathrm{N}$-terminal (NT) pro hormone BNP (NT-proBNP) are elevated, leading to hyperinflammation in epithelial cells in the airways. $^{3}$ High inflammation in such cells reduces antiviral immune response; hence the body allows the SARS-CoV-2 virus to enter and replicate. The viral replication leads to increased levels of virus-linked pyroptosis and apoptosis, which triggers the inflammatory responses due to the activation of pro-inflammatory chemokines and cytokines. Besides, type 2 diabetes leads to an impaired innate and cell-mediated, as well as humoral immunity that increases the susceptibility to viral infections. ${ }^{16}$ The chronic hyperglycemia plays a crucial role in immune response and viral infections through inflammatory processes that lead to the activation of the monocytes and promotes the synthesis of proinflammatory markers like cytokines. The activation of pro-inflammatory cytokines aggravates tissue inflammation and injury and worsens viral infections. Furthermore, hyperglycemia facilitates viral infections by increasing the expression of TNF- $\alpha$ and IL- 6 that further worsens inflammation. This inflammation occurs due to the activation of macrophages in the adipose tissues, which impairs the innate immune response, therefore favouring the invasion and virulence of viral infections, like SARS-CoV-2 or COVID-19 infections. ${ }^{16}$ People with type 2 diabetes present abnormal complement activation and dysfunctional type IV or delayed hypersensitivity reaction that hinders immune response. Such activity may cause endothelial dysfunction that enables easier access of viruses like SARS-CoV-2 virus into the host cells. ${ }^{17,18}$

Interferon and interleukins also have antiviral activity and act by reducing chronic inflammation. However, in type 2 debates, interleukins production is reduced, and type 1 interferon and IL-22 are suppressed; hence, antiviral activity reduces while the inflammation process is also enhanced. This affects the normal functioning of the immune system or causes immune defects, which enhance the ability of viruses to enter host cells. ${ }^{19,20}$
Diabetes mellitus is a chronic inflammatory health condition characterized by various vascular and metabolic abnormalities affecting body response to pathogens. Insulin resistance and hyperglycemia facilitate increased production of glycosylation end product and synthesis of pro-inflammatory cytokines. ${ }^{15}$ It also stimulates the synthesis of adhesion molecules, which mediate tissue inflammation. This tissue inflammation process has an underlying mechanism that causes a high propensity to viral infections in patients with type 2 diabetes. ${ }^{15}$ Diabetes mellitus enhances viral replication in the body due to an impaired immune system. People with type 2 diabetes have impaired beta-cell responsiveness, insulin resistance, and poor metabolism of glucose. This enhances persistent viral infections that led to cell damage. ${ }^{14}$ it is evident that people with type 2 diabetes are also highly prone to viral infections due to compromised antiviral immune response system. In type 2 diabetes, the production or release of cytokines, like IFN- $\alpha$, is reduced due to damages to the natural barriers. Damaging natural barriers leads to delayed induction of IFN- $\alpha$ and the production of IgG and IgM antibodies, which are the essential antibodies to fight against the invasion of viruses. ${ }^{15}$ Innate and adaptive immune systems help protect the human against viral infections. However, in type 2 diabetes, both innate and adaptive immune systems are compromised due to defects in the phagocytosis, dysfunction of immune cells, and suppression of cytokines. ${ }^{4}$ The compromised innate and adaptive systems promote viral entry into the body and facilitate replication of the virus in the body. ${ }^{5}$ One of the current viral infections that affect people with type 2 diabetes is COVID-19 infection. In type 2 diabetes, the innate and adaptive systems are compromised due to impaired production of IL-2, IL-6, IL-10, and poor functioning of T-cells, hence reducing the production of IFN- $\gamma$ and TNF- $\alpha$. Impairment in such immune systems makes the body inactive to fight the virus. This facilitates viral entry of SARS-CoV and MERS-CoV. ${ }^{16}$

Besides, people with type 2 diabetes mellitus are highly prone to viral infections due to over activated inflammatory responses that impair innate and adaptive immune systems. Such patients have high levels of inflammatory markers, like CRP, LDH, and troponin, leading to hyperinflammation in the epithelial cells. Inflammation of epithelial cells impairs antiviral response and makes the body susceptible to viral infections. Impaired antiviral immune responses facilitate the viral entry of the SARS-CoV-2 virus and other viruses to replicate and grow within body tissues and cause deaths. ${ }^{16}$ The viral replication inside the body of host cells of a patient with type 2 diabetes mellitus promotes viruslinked pyroptosis and apoptosis, leading to activation of pro-inflammatory chemokines and cytokines. Thus, the pro-inflammatory process triggers inflammatory responses that cause dysfunction or compromised innate and humoral immunity and makes the body highly susceptible to viral infections. ${ }^{16}$ Besides, hyperinflammation activates monocytes and exacerbate 
tissue inflammation that facilitates viral infections due to increased expression of pro-inflammatory markers, such as IL- 6 and TNF- $\alpha$. The inflammatory responses activate macrophages located in the adipose tissues, and such activation impairs the innate immune response, therefore favouring virulence and viral invasion. ${ }^{16}$

There are also reported cases of delayed or dysfunctional type $1 \mathrm{~V}$ hypersensitivity reaction in type 2 diabetes mellitus that hinder the innate or adaptive immune response. The impaired immune response causes dysfunction of endothelial cells, which facilitates the entry SARS-CoV-2 into the body of a person with type 2 diabetes. ${ }^{17,18}$

People with type 2 diabetes have compromised innate and adaptive immune response that facilitates viral infections. A compromised immune system indicates that the body of such patients cannot fight against infections, hence provides the basis for viruses to enter and replicate in the host cells. Consequently, it is essential for patients with type 2 diabetes to seek treatment to boost their immune system to reduce viral infections.

\section{CONCLUSION}

Type 2 diabetes is a primary medical problem and has become among the leading cause of death globally. Strong evidence points to the impacts of type 2 diabetes on immune response and viral infections. Notably, the susceptibility of people with type 2 diabetes to viral infections is associated with immune defects or the inability of immune systems to fight the pathogens. In type 2 diabetes mellitus, impaired beta-cell responsiveness, high glucose concentrations, and cytokine production impairment are involved in viral infections. Clinical evidence supports that the innate and adaptive immune system response is altered through such mechanisms, which facilitates pathogenic invasion of viruses like HHV-8, SARS-CoV, MERS-CoV, SARS$\mathrm{CoV}-2$, and hepatitis $\mathrm{C}$ virus. It is apparent that type 2 diabetes suppresses immune cells; therefore, the body cannot fight any microbes, which allows for viral replication and progression in the host cells.

Funding: No funding sources Conflict of interest: None declared Ethical approval: Not required

\section{REFERENCES}

1. Ferlita S, Yegiazaryan A, Noori N, Lal G, Nguyen T, To $\mathrm{K}$, et al. Type 2 diabetes mellitus and altered immune system leading to susceptibility to pathogens, especially Mycobacterium tuberculosis. J Clin Med. 2019;8(12):2219.

2. Zhou T, Hu Z, Yang S, Sun L, Yu Z, Wang G. Role of adaptive and innate immunity in type 2 diabetes mellitus. J Diabetes Res. 2018;1:1-9.
3. Erener S. Diabetes, infection risk and COVID-19. Mol Metab. 2020;39:101044.

4. Berbudi A, Rahmadika N, Tjahjadi AI, Ruslami R. Type 2 diabetes and its impact on the immune system. Curr Diabetes Rev. 2020;16(5):442.

5. American Diabetes Association. Standards of medical care in diabetes-2013. Diabetes Care. 2013;36(1):S11-66.

6. Tanaka T, Narazaki M, Kishimoto T. IL-6 in inflammation, immunity, and disease. Cold Spring Harb Perspect Biol. 2014;6(10):a016295.

7. Wang X, Ota N, Manzanillo P, Kates L, ZavalaSolorio J, Eidenschenk C, et al. Interleukin-22 alleviates metabolic disorders and restores mucosal immunity in diabetes. Nature. 2014;514(7521):237-41.

8. Hu R, Xia CQ, Butfiloski E, Clare-Salzler M. Effect of high glucose on cytokine production by human peripheral blood immune cells and type I interferon signaling in monocytes: implications for the role of hyperglycemia in the diabetes inflammatory process and host defense against infection. Clin Immunol. 2018;195:139-48.

9. Chao WC, Yen CL, Wu YH, Chen SY, Hsieh CY, Chang TC, et al. Increased resistin may suppress reactive oxygen species production and inflammasome activation in type 2 diabetic patients with pulmonary tuberculosis infection. Microbes Infect. 2015; 17(3):195-204.

10. Jafar N, Edriss H, Nugent K. The effect of short-term hyperglycemia on the innate immune system. Am J Med Sci. 2016;351(2):201-11.

11. Restrepo BI, Twahirwa M, Rahbar MH, Schlesinger LS. Phagocytosis via complement or Fc-gamma receptors is compromised in monocytes from type 2 diabetes patients with chronic hyperglycemia. PloS One. 2014;9(3):e92977.

12. Pavlou S, Lindsay J, Ingram $\mathrm{R}, \mathrm{Xu} \mathrm{H}$, Chen $\mathrm{M}$. Sustained high glucose exposure sensitizes macrophage responses to cytokine stimuli but reduces their phagocytic activity. BMC Immunol. 2018;19(1):24.

13. Berrou J, Fougeray S, Venot M, Chardiny V, Gautier JF, Dulphy N, et al. Natural killer cell function, an important target for infection and tumor protection, is impaired in type 2 diabetes. Plos One. 2013;8(4):e62418.

14. Casqueiro J, Casqueiro J, Alves C. Infections in patients with diabetes mellitus: A review of pathogenesis. Indian $\mathrm{J}$ Endocrinol Metab. 2012;16(1):S27.

15. Kumar M, Roe K, Nerurkar PV, Namekar M, Orillo $\mathrm{B}$, Verma $\mathrm{S}$, et al. Impaired virus clearance, compromised immune response and increased mortality in type 2 diabetic mice infected with West Nile virus. PLoS One. 2012;7(8):e44682.

16. Milionis C, Milioni SO. A brief analysis and hypotheses about the risk of COVID-19 for people with type 1 and type 2 diabetes mellitus. J Diabetes Metab Disord. 2020:1-5. 
17. Sardu C, Gambardella J, Morelli M, Wang X, Marfella R, Santulli G. Is COVID-19 an endothelial disease? Clinical and basic evidence. J Clin Med. 2020;9(5):1417.

18. Hussain A, Bhowmik B, do Vale Moreira NC. COVID-19 and diabetes: Knowledge in progress. Diabetes Res Clin Pract. 2020;162:1-9.

19. Daryabor G, Atashzar MR, Kabelitz D, Meri S, Kalantar K. The effects of type 2 diabetes mellitus on organ metabolism and the immune system. Front Immunol. 2020;11:1-22.
20. Toniolo A, Cassani G, Puggioni A, Rossi A, Colombo A, Onodera T, et al. The diabetes pandemic and associated infections: suggestions for clinical microbiology. Clin Microbiol Rev. 2019;30(1):1-17.

Cite this article as: Ambati SK. Compromised immune response to viral infections in type 2 diabetes mellitus. Int J Community Med Public Health 2020;7:5203-7. 